\title{
Polypoidal choroidal vasculopathy and exudative age- related macular degeneration in Greek population
}

ID Ladas', AA Rouvas', MM Moschos², EE Synodinos ${ }^{1}$, DA Karagiannis ${ }^{1}$ and CN Koutsandrea ${ }^{1}$

\begin{abstract}
Purpose To study the prevalence, the clinical features, and the visual prognosis without treatment of polypoidal choroidal vasculopathy (PCV) in a large series of Greek patients presenting with exudative maculopathy.

Methods The medical records, photographs, as well as fluorescein and indocyanine green (ICG) angiograms of a series of 268 consecutive elderly white Greek patients, who were originally diagnosed as having exudative age-related macular degeneration (AMD) were reviewed retrospectively.
\end{abstract}

Results In all, 22 of the $268(8.2 \%)$ patients initially suspected of having AMD were ultimately diagnosed with PCV. In 15 of the 22 (68.2\%) patients with PCV, the polypoidal lesions were located in the peripapillary area. Large soft drusen were present in only two fellow eyes of the $10(20 \%)$ patients with unilateral PCV compared with 120 fellow eyes of the $148(81.1 \%)$ patients with unilateral AMD. At the last examination, 11 of the 22 (50\%) patients with PCV and 120 of the 246 (48.8\%) patients with AMD presented a visual acuity of less than $6 / 60$ in at least one eye due to scar formation in the macula.

Conclusions PCV is not an infrequent disease in Greece. A measurable number of Greek patients with findings suggestive of exudative AMD will instead have PCV. ICG angiography is important in differentiating between these two clinical entities. In Greeks, polypoidal lesions are predominately peripapillary and are not usually associated with macular drusen in the fellow eye. PCV and exudative AMD do not differ significantly in terms of their natural course and visual prognosis in Greek patients. Eye (2004) 18, 455-459. doi:10.1038/sj.eye.6700706
Keywords: polypoidal choroidal vasculopathy; exudative age-related macular degeneration

\section{Introduction}

Since about two decades, polypoidal choroidal vasculopathy (PCV) has been recognized as a new exudative disorder of the macula. ${ }^{1-17}$ While its pathogenesis remains unclear, it is generally thought to be an inner choroidal vascular abnormality characterized by two main components: (1) a peculiar network of fine branching vessels external to choriocapillaris and (2) multiple terminal aneurysmal dilations at the border of the vascular network. These vascular dilations are sometimes visible clinically as a reddish-orange spheroidal polypoidal lesions, particularly if they are large enough and there is sufficient atrophy of the overlying retinal pigment epithelium (RPE). It is generally accepted that indocyanine green (ICG) angiography is the examination of choice for the identification of the vascular polypoidal lesions of the PCV with great sensitivity and specificity. ${ }^{2,4-6,11,14}$

In the past, PCV has been typically described as a bilateral peripapillary disorder of middleaged hypertensive black females. ${ }^{1-3,5}$ However, recent studies report that it also occurs in individuals of all races, ethnic groups, and sexes with a broad age range and that it appears to share similar characteristics with exudative age-related macular degeneration (AMD). ${ }^{8,9,11,12,14}$ Within the expanded clinical spectrum of PCV, there is a possibility that some patients who were originally diagnosed with exudative AMD may actually have exudative maculopathy secondary to $\mathrm{PCV}$ rather than to AMD.
${ }^{1}$ Department of Ophthalmology Medical School of Athens University Athens, Greece

${ }^{2}$ Jules Gonin Eye Hospital University of Lausanne Switzerland

Correspondence: ID Ladas 8 Meg. Alexandrou Str. GR-152 36 P

Penteli, Athens, Greece Tel: + 30108032338

Fax: + 30106130351

E-mail:

ladas@ath.forthnet.gr

Received: 8 October 2002 Accepted: 21 March 2003 
The purpose of this retrospective study was to establish the prevalence, the clinical features, as well as the visual prognosis without treatment of PCV in a large consecutive series of white Greek patients presenting with exudative maculopathy, who were originally diagnosed as having exudative AMD.

\section{Materials and methods}

We reviewed retrospectively the medical records, colour and/or red-free photographs, as well as fluorescein and ICG angiograms on a series of 268 consecutive white Greek patients, who were referred to us with the initial diagnosis of exudative AMD during the last 2 years. All patients were older than 50 years of age and had a complete ocular history, slit-lamp evaluation, and posterior fundus examination. Fluorescein angiography with $5 \mathrm{~cm}^{3}$ of $10 \%$ solution and ICG angiography with a $50 \mathrm{mg}$ dose had been performed in both eyes of each patient through a retinal camera (Topcon TRC-50IA, Topcon, Tokyo, Japan), with images digitally stored and processed (Topcon IMAGEnet H-1024 Digital Imaging System, Topcon, Tokyo, Japan).

PCV was diagnosed when one or more polypoidal dilations in the inner choroid were seen on ICG angiography as areas of early, intense hyperfluorescence. The diagnosis was made by two masked retinal specialists (AAR and CNK). Only if there was uncertainties in interpretation, the decision was referred to the lead author (IDL).

Patients were excluded if they had other forms of neovascularized maculopathy, such as pathologic myopia, angioid streaks, presumed ocular histoplasmosis syndrome, and central serous chorioretinopathy, or opacifications of the media that precluded a clear fundus or angiographic examination.

The two-tailed Fisher's exact test and the two-tailed unpaired $t$-test were used for data analysis. A $P$-value of less than 0.05 was considered to be statistically significant.

\section{Results}

In total, 22 of the 268 (8.2\%) patients initially suspected of having exudative AMD were ultimately diagnosed with PCV. Of the 246 patients, 125 (50.8\%) with AMD and 13 (59\%) of the 22 patients with PCV were male. The

difference in sex prevalence between the two groups was not statistically significant $(P=0.5098)$. The patients with PCV (mean age: 72.5 years, range $51-89$ years, SD: 8.12 ) were significantly younger than those with AMD (mean age: 77 years, range $50-90$ years, SD: 7.02) $(P=0.0048)$. Eight of the $22(36.4 \%)$ patients with PCV and 151 of the $246(61.4 \%)$ patients with AMD had a history of hypertension $(P=0.0392)$. The difference between the two groups was statistically significant.

The percentage of bilateral involvement was greater in the patients with PCV $(54.5 \%)$ than in those with AMD $(39.8 \%)$. However, the difference between the two groups was not statistically significant $(P=0.1844)$. In 15 of the $22(68.2 \%)$ eyes with PCV, the polypoidal lesions were located in the peripapillary area (Figure 1), while only nine of the $246(3.7 \%)$ eyes with AMD presented a peripapillary $\mathrm{CNV}$.

In more than one-third (36.3\%) of the eyes with PCV, the polypoidal lesions were associated with a large PED ( $>2$ disc diameters) (Figure 2 ). In eyes with AMD, the frequency of PED was statistically significant lower $(10.5 \%)(P=0.1860)$. Large soft drusen (diameter $>63 \mu \mathrm{m}$ ) were observed in only two fellow eyes of the 10 (20\%) patients with unilateral PCV compared with 120 fellow eyes of the $148(81.1 \%)$ patients with unilateral AMD. The difference between the two groups was extremely significant $(P=0.0001)$.

At the last examination, 11 of the $22(50 \%)$ patients with PCV and 120 of the $246(48.8 \%)$ patients with AMD presented a best corrected visual acuity of less than 6/60 in at least one eye due to scar formation in the macula (Figure 1).

\section{Discussion}

The expanding clinical spectrum of PCV is still an object of continuous investigation. While in the past, some reports exclusively associated PCV with old black females patients, ${ }^{1-3,5}$ recently it has also been identified in male patients, other races, and ethnic groups. $8,9,11,12,14,15$ Curiously in Japanese and Chinese patients, PCV is evident more prevalent in men. ${ }^{8,15}$ In our series of white Greek patients, the difference in sex prevalence between PCV and AMD was not statistically significant. Further, the frequency of PCV was almost the same in both sexes.

Although our patients with PCV were younger than those with AMD, the age cannot be used as a criterion for the differentiation between these two clinical entities, as all the patients of our series were older than 50 years of age.

A measurable number of our patients referred to us with the initial diagnosis of exudative AMD were ultimately diagnosed as having PCV. The role of ICG angiography in differentiating between PCV and AMD was decisive. With ICG angiography, we could identify the polypoidal vascular lesions and distinguish them from the typical forms of CNV (classic or occult) with great sensitivity and specificity (Figures 1 and 2). Moreover, many of our patients with PCV presented polypoidal vascular lesions, which were not detectable 

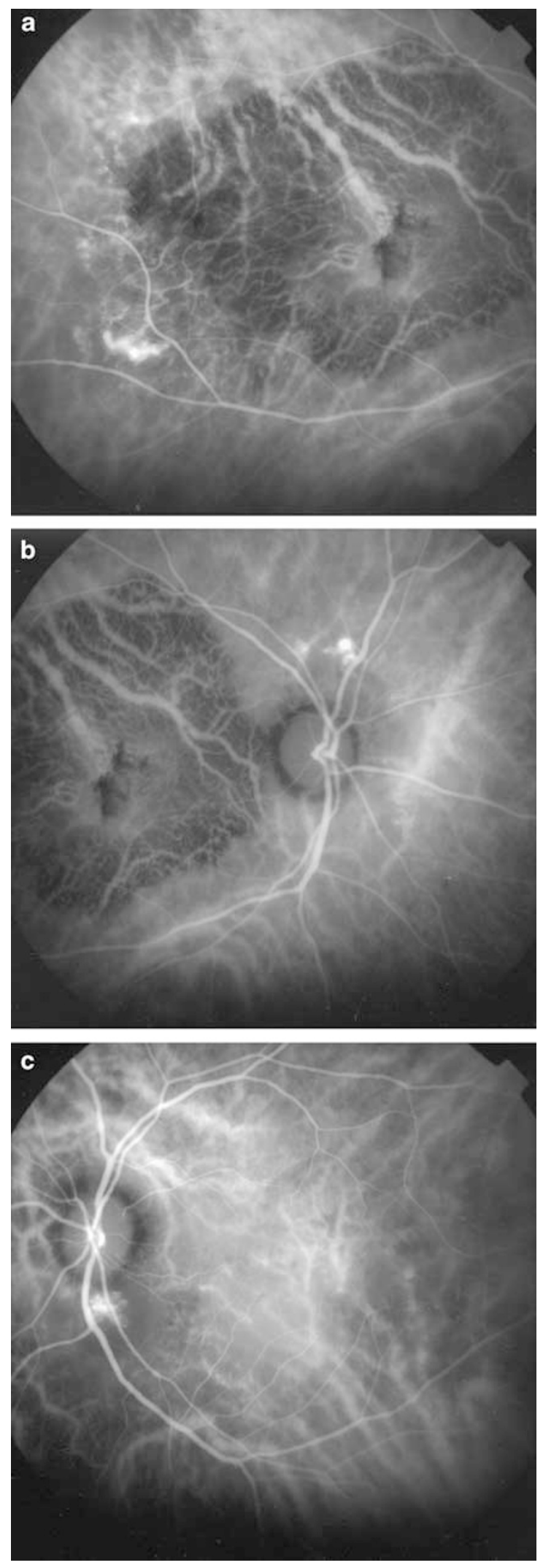

either ophthalmoscopically or with fluorescein angiography (Figure 2).

Previous reports, including one of describing the histopathologic changes of the $P C V,{ }^{10}$ have emphasized the peripapillary location of the polypoidal lesions and suppose a peripapillary choroidal origin of the vascular abnormalities. ${ }^{1,24}$ More recent studies showed that polypoidal lesions can be identified, apart from the peripapillary area, in the macula or less frequently in the periphery of the fundus. ${ }^{5,7-9,11,15}$ Our results suggested a peripapillary predominance of the location of the polypoidal lesions in Greeks (Figure 1). However, in a not insignificant number of our patients, the polyps were located in the macula associated with or no peripapillary lesions.

Our results along with others ${ }^{9,14,15}$ showed a lower incidence of large soft drusen in the fellow eye of patients with PCV compared with their counterparts with exudative AMD. In our series of white Greek patients, the difference between the two groups was statistically extremely significant.

Using the ICG angiography, Ahuja et $a l^{11}$ detected a high proportion $(85 \%)$ of patients with PCV in a largely white patient population with haemorrhagic and exudative PEDs. In our series of homogeneous white population in Greece, the frequency of PED was significantly higher in patients with PCV (36.3\%) than in those with exudative AMD (only 10.5\%). The cause of the frequent presence of a large PED in patients with PCV is unknown. We suppose that leakage from the large inner choroidal polypoidal vascular lesions may explain the predilection for PED formation in patients with PCV, as well as the location of the PED contiguously to the polypoidal lesions.

The visual prognosis of PCV without treatment is not well known. It is possible that, because of the initial exudative lesions sparing the foveal avascular zone, the patients are essentially asymptomatic probably for a prolonged period. Yannuzzi et $a l^{9}$ reported that the natural course of the disease 'seems to be favourable' as several patients have chronic multiple recurrent serosanguineous detachments of the RPE and neurosensory retina with long-term preservation of good central vision. A favourable course has also been reported for Japanese patients with PCV. ${ }^{8}$ However, a guarded prognosis of PCV has been suggested for patients of other races and ethnic groups. ${ }^{12,15}$ Accordingly, the natural course of PCV seems to be different in different races and ethnic groups.

Figure 1 Early phase ICG angiograms of a 70-year-old man with PCV. (a) Polypoidal lesions just temporal to a large chorioretinal scar in the macula of the right eye. $(b, c)$ Peripapillary polypoidal lesions in the right (b) and left (c) eye. 

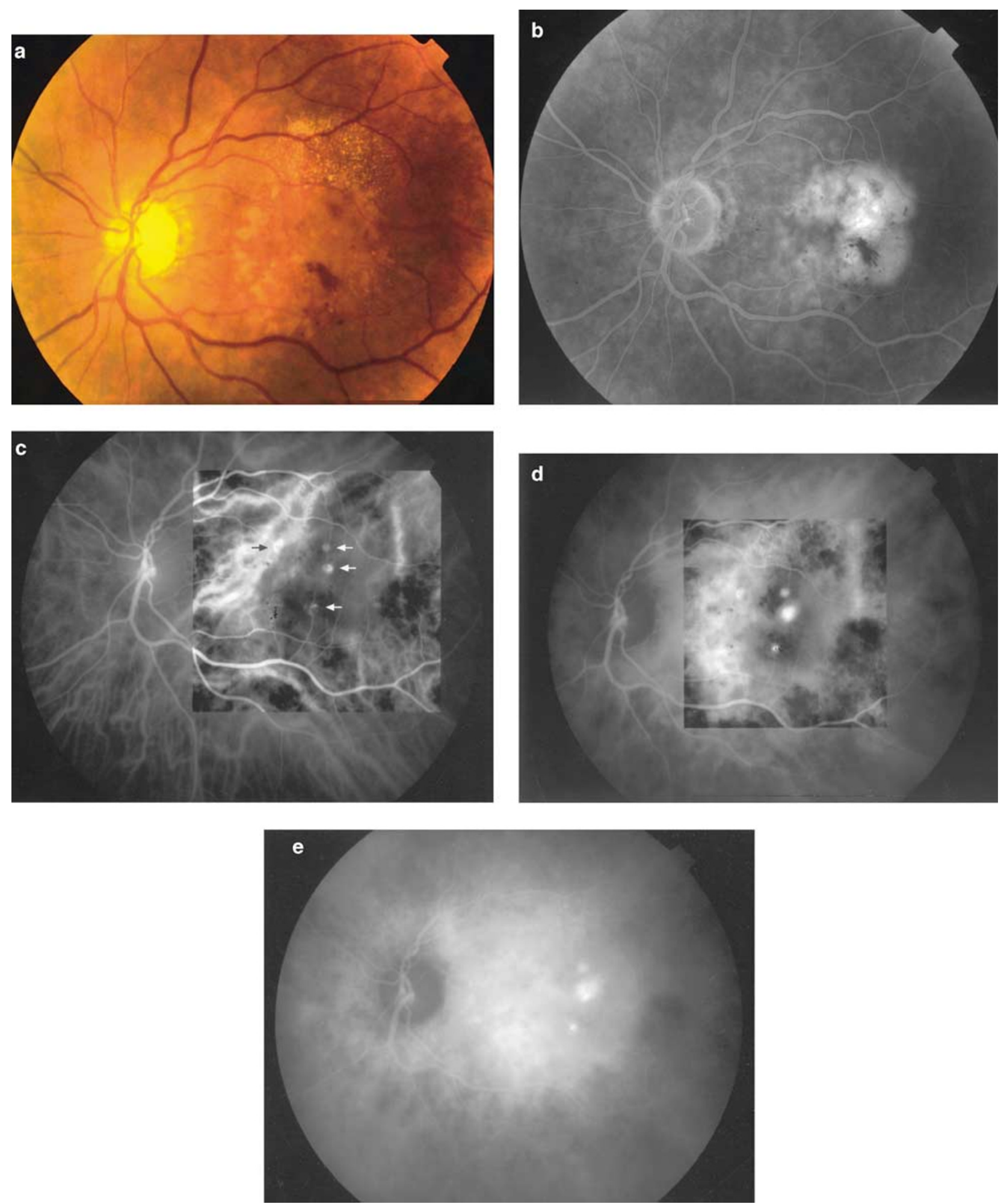

Figure 2 PCV in a 53-year-old woman. (a) Colour fundus photograph shows exudative maculopathy. (b) Fluorescein angiogram shows a large PED of the macula. (c-e) ICG angiograms reveal active (white arrows) and inactive (black arrow) macular polypoidal vascular lesions associated with PED. 
In our series, half of our Greek white patients with PCV presented a visual acuity of less than 6/60 in at least one eye due to hypertrophic subretinal scar formation in the macula (Figure 1). Unlike AMD, fibrovascular disciform scarring is reported to be unusual in PCV. ${ }^{1}$ Why some eyes with PCV have spontaneous resolution of the exudative manifestations due to autoinfarction of the polypoidal complexes, while others lead to scar formation is unknown.

A very important reason to differentiate PCV from exudative AMD is that the patients with PCV are likely to require a different therapeutic approach, whether one considers conventional thermal laser $6,8,12,15,16$ or an alternative treatment approach such as photodynamic therapy with verteporfin, ${ }^{17}$ which is currently under investigation in our clinic. Some authors have reported favourable results of ICG-guided laser treatment of polypoidal lesions with a technique similar to that used for photocoagulation of a leaking retinal arterial macroaneurysm (mild thermal reaction). 6,8,12,17 The benefit of sparing the nerve-fibre layer, compared with the intense confluent burn that is produced from the direct heavy laser treatment on a CNV due to AMD, is very apparent in the visual field of the treated eye. It seems that after the ICG-guided mild laser treatment of the active polypoidal lesions, some eyes present a quick resolution of the exudative manifestations in the macula with low or no recurrences. If the latter will be confirmed by larger prospective randomized clinical trials with greater follow-up time, we believe that it would be the most important evidence for the usefulness of the differentiation between PCV and AMD in everyday clinical practice.

In conclusion, $\mathrm{PCV}$ is not an infrequent disease in Greece. As PCV and exudative AMD share similar exudative manifestations in the macula, a measurable number of Greek patients with findings suggestive of exudative AMD will instead have PCV. ICG angiography is the tool of choice in differentiating between these two clinical entities. Based on the data of our study, the diagnosis of PCV should be suspected in elderly Greek patients presenting exudative maculopathy with extramacular lesions, large PEDs, and few or no drusen in the fellow eye. Although there is a great variation in the natural course of PCV among different races and ethnic groups, the visual prognosis of the Greeks with PCV seems to be rather guarded. PCV and AMD do not differ significantly in terms of their natural course and visual prognosis in Greek patients.

\section{References}

1 Yannuzzi LA, Sorenson J, Spaide RF, Lipson B. Idiopathic polypoidal choroidal vasculopathy (IPCV). Retina 1990; 10: $1-8$.

2 Spaide RF, Yannuzzi LA, Slakter JS, Sorenson J, Orlach DA. Indocyanine green video angiography of idiopathic choroidal vasculopathy. Retina 1995; 15: 100-110.

3 Ross RD, Gitter KA, Cohen G, Schomaker KS. Idiopathic polypoidal choroidal vasculopathy associated with retinal arterial macroaneurysm and hypertensive retinopathy. Retina 1996; 16: 105-111.

4 Phillips II WB, Regillo CD, Maguire JI. Indocyanine green angiography of idiopathic polypoidal choroidal vasculopathy. Ophthalmic Surg Lasers 1996; 27: 467-470.

5 Yannuzzi LA, Ciardella A, Spaide RF, Rabb M, Freund KB, Orlock DA. The expanding clinical spectrum of idiopathic choroidal vasculopathy. Arch Ophthalmol 1997; 115: 478-485.

6 Gomez-Ulla F, Gonzalez F, Torreiro MG. Diode laser photocoagulation in idiopathic polypoidal choroidal vasculopathy. Retina 1998; 18: 481-483.

7 Moorthy RS, Lyon AT, Rabb MF, Spaide RF, Yannuzzi LA, Jampol LM. Idiopathic polypoidal choroidal vasculopathy of the macula. Ophthalmology 1998; 105: 1380-1385.

8 Uyama M, Matsubara T, Fukushima I, Matsunaga H, Iwashita K, Nagai Y et al. Idiopathic polypoidal choroidal vasculopathy in Japanese patients. Arch Ophthalmol 1999; 117: 1035-1042.

9 Yannuzzi L, Wong DWK, Sforzolini BS, Goldbaum M, Tang $\mathrm{KC}$, Spaide RF et al. Polypoidal choroidal vasculopathy and neovascularized age-related macular degeneration. Arch Ophthalmol 1999; 117: 1503-1510.

10 Lafaut BA, Aisenbrey S, Van den Broecke C, Bartz-Schmidt K-U, Heimann K. Polypoidal choroidal vasculopathy pattern in age-related macular degeneration: a clinicopathologic correlation. Retina 2000; 20: 650-654.

11 Ahuja RM, Stanga PE, Vingerling JR, Reck AC, Bird AR. Polypoidal choroidal vasculopathy in exudative and haemorrhagic pigment epithelial detachments. $\mathrm{Br} \mathrm{J}$ Ophthalmol 2000; 84: 479-484.

12 Lafaut BA, Leys AM, Snyers B, Rasquin F, De Laey JJ. Polypoidal choroidal vasculopathy in Caucasians. Graefes Arch Clin Exp Ophthalmol 2000; 238: 752-759.

13 Lip PL, Hope-Ross MW, Gibson JM. Idiopathic polypoidal choroidal vasculopathy: a disease with diverse clinical spectrum and systemic associations. Eye 2000; 14: 695-700.

14 Scassellati-Sforzolini B, Mariotti C, Bryan R, Yannuzzi LA, Giuliani M, Giovanini A. Polypoidal choroidal vasculopathy in Italy. Retina 2001; 21: 121-125.

15 Kwok AKH, Lai TYY, Chan CWN, Neoh E-L, Lam DSC. Polypoidal choroidal vasculopathy in Chinese patients. $\mathrm{Br} J$ Ophthalmol 2002; 86: 892-897.

16 Iida T, Yannuzzi LA, Freund KB, Ciardella AP, Costa DL, Huang SJ et al. Retinal angiopathy and polypoidal choroidal vasculopathy. Retina 2002; 22: 455-463.

17 Quaranta M, Mauget-Faysse M, Coscas G. Exudative polypoidal vasculopathy and photodynamic therapy with verteporfin. Am J Ophthalmol 2002; 134: 277-280. 